\title{
Generalized method of fundamental solutions (GMFS) for boundary value problems
}

\author{
J.J. Yang ${ }^{1}, \quad$ J.L. Zheng ${ }^{1 *}, \quad$ P.H. Wen ${ }^{2 *}$ \\ ${ }^{1}$ School of Traffic and Transportation Engineering, Changsha University of Science and \\ Technology, Changsha, 410114, China \\ ${ }^{2 *}$ School of Engineering and Materials Science, Queen Mary, University of London, London E1 \\ $4 N S, U K$
}

*Corresponding author: p.h.wen@qmul.ac.uk (Wen)

In order to cope with the instability of the method of fundamental solutions (MFS), which caused by source offset, or source location, or fictitious boundary, a generalized method of fundamental solutions (GMFS) is proposed. The crucial part of the GMFS is used a generalized fundamental solution approximation (GFSA), which adopts a bilinear combination of fundamental solutions to approximate, rather than the linear combination of the MFS. Then the numerical solution of the GMFS is decided by a group of offsets corresponding to an intervention-point diffuse (IPD), instead of the MFS' only offset of a single source. To demonstrate the effectiveness of the proposed approach, four numerical tests are given. The results have shown that the GMFS is more accurate, stable, and better convergence than the traditional MFS.

Key words: meshless methods, boundary methods, method of fundamental solutions (MFS), offset, fictitious boundary, intervention point. 


\section{Introduction}

In recent years the method of fundamental solutions (MFS), a boundary meshless method, has attracted great attention for solving homogeneous differential equations [1-9]. The MFS is quite simple, efficient and easy for implementation, and has avoided the singular integrals which is necessary in certain boundary meshless methods, such as BNM [10], LBIE [11], HBNM [12], BCM [13], BFM [14]. Furthermore, it could be highly accurate and convergent rapidly when an appropriate offset is selected [15].

However, despite the effectiveness and simplicity of the MFS, there are still some outstanding theoretical and numerical issues to be addressed [16-18]. One of the main issues yet to be resolved is the optimal choice of the offset. In the MFS, a fictitious boundary outward offset to the real boundary with a distance parameter $d$ is required in order to define the source points outside the domain. The offset $d$ is sensitive and vital to the accuracy of the MFS. It is possible that we could set a reasonable range for the offset based on the experience. However, it is not always effective, because a good offset for a certain problem could be bad for another problem. Despite the intensive research, this "offset dilemma" has been an outstanding research topic in the MFS $[19,20]$.

In the past, various approaches have been proposed to alleviate this difficulty in the MFS such as the BKM [21-23] and BCM [24, 25]. Instead of using the singular fundamental solutions as used in the MFS, these methods use a non-singular kernels or general solutions. As such, the source points can be located on the physical/real boundary, and the fictitious boundary is not needed. However, it is difficult to find the non-singular kernels or general solutions for some practical problems. Even though the non-singular kernels or general solutions can be found, the accuracy is normally not very impressive.

Another proposed method worthy to mention is the non-singular method of fundamental solution (NMFS) [26, 27]. For the method, a desingularization technique is used to regularize the singularity of the fundamental solution, so the source points can be located at the real boundary, and the fictitious boundary is not necessary. Nevertheless, the boundary nodes should be distributed regularly, an available desingularization for arbitrary problems is still a problem, and the tedious desingularization procedure is somewhat at the expense of its simplicity.

The singular boundary method (SBM) [28-32] uses an origin intensity factor (OIF) to substitute the singularity. In this way, the source points can be located on the physical boundary, and the fictitious boundary is thus not necessary. But, the method should decide the OIFs which is not trivial, and the given problem should be solved twice.

Moreover, a boundary distributed source (BDS) method [33] should be noticed too. For the 
BDS method, the source points may not be offset, but should be distributed. The singular fundamental solution is integrated firstly over the distributed source which covering the source points. If the distributed source with a simple shape, such as a circle, then the singular integrals could be evaluated analytically. However, the singular integrals are not always analytical, and the solution is inaccurate near the boundary regions. An improved BDS method $[34,35]$ uses a boundary-integral technique to determine the singular integrals. But, it is necessary to directly calculate the singular integrals, correspondingly.

In some sense the above mentioned efforts overcome the old challenge of the MFS at a price of creating more or less new problems. From our understanding, the tenacious barrier of the MFS is still open for efforts. So, we will try to give another option for the issue of the MFS.

\section{Generalized fundamental solution approximation}

The MFS uses a fundamental solution approximation (FSA), which was first proposed by Kupradze and Aleksidze [36-38], as the basis function for solving homogeneous equations. It is notable that, another independent work with the same concept was also proposed by Wen [39] which is called the point intensity method (PIM). Let $u(\boldsymbol{x})$ be a field variable in a given domain $\Omega$ bounded by $\Gamma$. The basic ideas of FSA is to express $u(\boldsymbol{x})$ as a linear combination of fundamental solutions:

$$
u(\boldsymbol{x})=\sum_{J=1}^{N} a_{J} \psi_{J}(\boldsymbol{x}, \boldsymbol{s}), \quad \boldsymbol{x} \in \bar{\Omega},
$$

where $\psi_{J}(\boldsymbol{x}, \boldsymbol{s}) \equiv \psi\left(\boldsymbol{x}, \boldsymbol{s}_{J}\right) \equiv \psi\left(r_{J}\right)$ is the fundamental solution, $r_{J}=\left\|\boldsymbol{x}-\boldsymbol{s}_{J}\right\|_{2}$ the Euclidean norm between the measuring point $\boldsymbol{x}$ and the source point $s_{J}, a_{J}$ the intensity coefficient ats $_{J}$, and $\bar{\Omega} \equiv \Omega \mathrm{U} \Gamma$.

Being different from the FSA, the generalized fundamental solution approximation (GFSA) uses a bilinear combination of fundamental solutions to approximate $u(\boldsymbol{x})$ as follows:

$$
u(\boldsymbol{x})=\sum_{J=1}^{N} \sum_{p=1}^{N_{p}} a_{J} \psi_{p}^{J}(\boldsymbol{x}, \boldsymbol{s}), \quad\left\{\boldsymbol{s}_{p}\right\} \in \Omega_{J},
$$

where $\left\{\boldsymbol{s}_{p}\right\} \notin \bar{\Omega}$ is the intervention-point diffuse (IPD) of the source node $\boldsymbol{x}_{J}[40], N_{p}$ its point number, $\Omega_{J}$ the diffuse domain centered at $\boldsymbol{x}_{J}$ which is sheared off by the boundary $\Gamma$, as shown in Fig.1 (a). Note that we use a superscript " $J$ " in the function $\psi$ to denote a correspondence with the source node $\boldsymbol{x}_{J}$. 

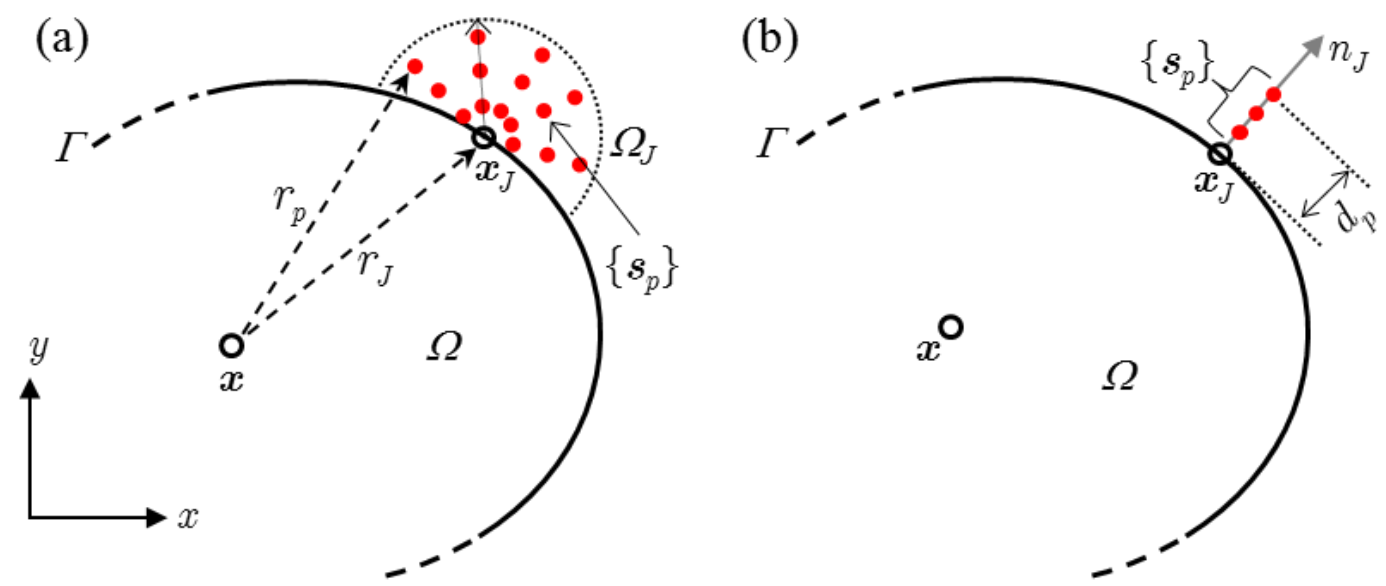

Fig. 1. Schematics of the GFSA: (a) Regional IPD; (b) Linear IPD.

Note that the diffuse domain $\Omega_{J}$ could be arbitrary selected outside the domain. For efficiency, we can also linearly diffuse the IPD, such as $\left\{\boldsymbol{s}_{p}\right\} \in n_{J}$, as shown in Fig. 1(b) where $n_{J}$ is the outward normal at $\boldsymbol{x}_{J}, d_{p}$ is the offset of an intervention point $\boldsymbol{s}_{p}$, as

$$
d_{p}=\left\|\boldsymbol{s}_{p}-\boldsymbol{x}_{J}\right\|_{2}, \quad p=1,2, \mathrm{~L}, N_{p} .
$$

In this paper, a diffuse scheme to choose $\left\{\boldsymbol{s}_{p}\right\} \in n_{J}$ is our focus. The appropriate choice of $N_{p}$ ( $\geq 5$ is suggested) and offsets $\left\{d_{p}\right\}$ is necessary. Tentatively, we choose $N_{p}=5$, and

$$
\begin{aligned}
& \left\{d_{p}\right\}=\left(\begin{array}{llll}
0.3, & 0.5, \quad 0.7, \quad 0.9, & 1.2
\end{array}\right) \cdot \bar{R}, \quad \text { for an outer boundary, }
\end{aligned}
$$

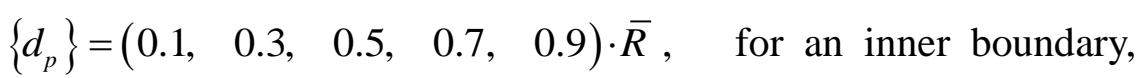

where $\bar{R}$ is defined as the normalized parameter for the boundary dimension

$$
\bar{R}=\frac{1}{\sqrt{D}}\left\|\frac{\max \left(x_{i}\right)-\min \left(x_{i}\right)}{2}\right\|_{2}
$$

in which the subscript " $i$ " is the component signal of the coordinates, $D$ is the number of dimensions. By default, the selection of $\left\{d_{p}\right\}$ in Eqs. (4) and (5) will be applied in the later section of numerical tests.

Obviously, when $N_{p}=1$, the GFSA is equivalent to the FSA. In other words, the GFSA is a generalized FSA. So we use a term of "generalized" to denote the novel approximation and the corresponding numerical method. Furthermore, the concept of the GMFS just fit the spirit of a saying, "Don't put all your eggs in the same basket".

\section{Generalized method of fundamental solutions (GMFS)}

Consider the following Laplace equation in a $2 \mathrm{D}$ domain $\Omega$ bounded by $\Gamma$ : 


$$
\nabla^{2} u(x)=0, \quad x \in \Omega,
$$

subject to boundary conditions (BCs):

$$
\begin{gathered}
u(\boldsymbol{x})=\bar{u}(\boldsymbol{x}), \quad \boldsymbol{x} \in \Gamma_{u}, \\
u_{, n}(\boldsymbol{x}) \equiv \frac{\partial u}{\partial n}(\boldsymbol{x})=\bar{q}(\boldsymbol{x}), \quad \boldsymbol{x} \in \Gamma_{t},
\end{gathered}
$$

where $\Gamma_{u}$ is the Dirichlet boundary, $\Gamma_{t}$ the Neumann boundary, $\Gamma=\Gamma_{u} \mathrm{U} \Gamma_{t}$, $\Gamma_{u} \mathrm{I} \Gamma_{t}=\varnothing, n$ the outward normal of the boundary, and $\bar{u}$ and $\bar{q}$ are the known functions on the boundary. The fundamental solution $\psi$ for the Laplacian is given by:

$$
\psi(r)=\left\{\begin{array}{cc}
\frac{-1}{2 \pi} \ln (r), & 2 D, \\
\frac{1}{4 \pi r}, & 3 D .
\end{array}\right.
$$
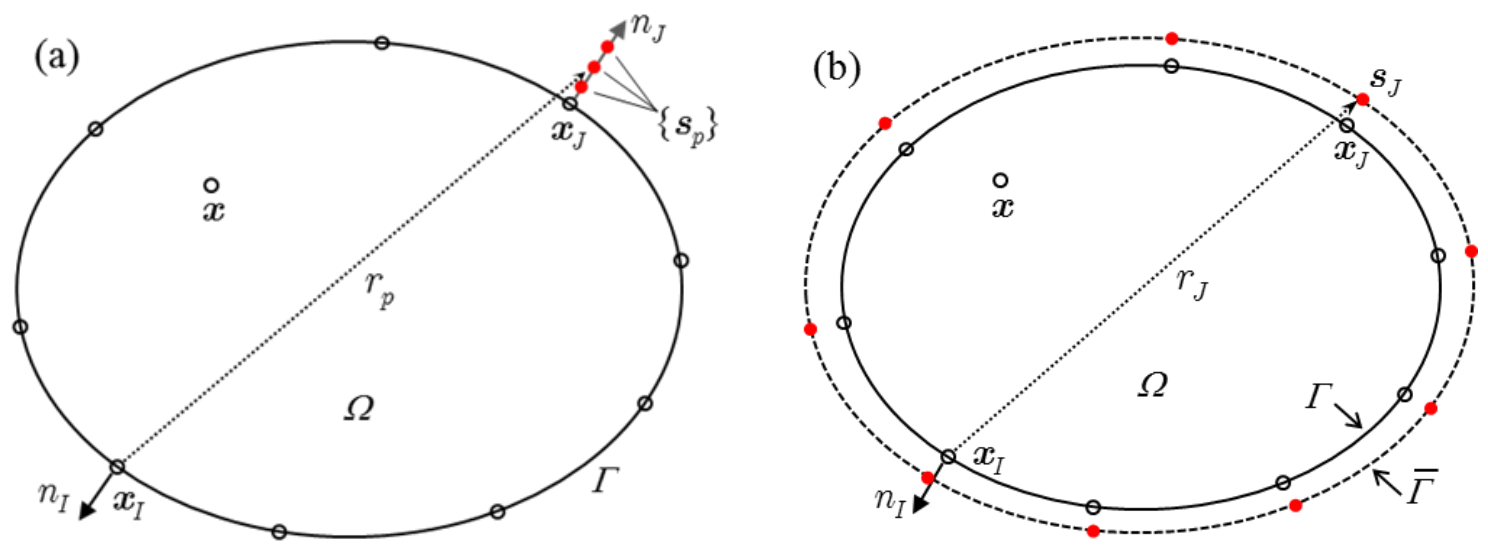

Fig. 2. Schematics of the GMFS and the MFS: (a) GMFS; (b) MFS.

The configuration of IPD (source-point cloud) of the GMFS is shown in Fig. 2(a). In contrast, the sources of the MFS are shown in Fig. 2(b). The target node $x_{I}$ is indexed for constructing the discrete system equations. We will first try to use the variation method. The functional variation is given as

$$
\delta \Pi_{2}=\sum_{x_{I} \in \Gamma_{u}} \delta u(u-\bar{u})+\sum_{x_{I} \in \Gamma_{t}} \delta u_{, n}\left(u_{, n}-\bar{q}\right) .
$$

Let $\delta \Pi_{2}=0$, then the BCs given by Eqs. (8) and (9) are satisfied. Then a GMFS1-type system of equations is obtained

$$
\bar{K} \boldsymbol{a}=\overline{\boldsymbol{F}}
$$

where $\overline{\boldsymbol{K}}$ is the whole "stiffness" matrix, $\overline{\boldsymbol{F}}$ the whole "load" matrix, and $\boldsymbol{a}=\left[a_{1}, a_{2}, \mathrm{~L}, a_{N}\right]^{T}$ the unknown intensity coefficients introduced in Eq. (2). Furthermore, 


$$
\overline{\boldsymbol{K}}=\boldsymbol{K}^{T} \boldsymbol{K}, \quad \overline{\boldsymbol{F}}=\boldsymbol{K}^{T} \boldsymbol{F}
$$

where

$$
\begin{gathered}
\boldsymbol{K}_{I J}= \begin{cases}\sum_{p=1}^{N_{p}} \psi_{p}^{J}\left(\boldsymbol{x}_{I}, \boldsymbol{s}\right), & \boldsymbol{x}_{I} \in \Gamma_{u}, \\
\sum_{p=1}^{N_{p}} \frac{\partial \psi_{p}^{J}\left(\boldsymbol{x}_{I}, \boldsymbol{s}\right)}{\partial n}, & \boldsymbol{x}_{I} \in \Gamma_{t},\end{cases} \\
\boldsymbol{F}_{I}= \begin{cases}\bar{u}\left(\boldsymbol{x}_{I}\right), & \boldsymbol{x}_{I} \in \Gamma_{u}, \\
\bar{q}\left(\boldsymbol{x}_{I}\right), & \boldsymbol{x}_{I} \in \Gamma_{t} .\end{cases}
\end{gathered}
$$

On the other hands, we can also directly solve the potential problems using the collocation method, and then a GMFS2-type system equation is obtained

$$
\boldsymbol{K} \boldsymbol{a}=\boldsymbol{F}
$$

where the "stiffness" matrix $\boldsymbol{K}$ and the "load" matrix $\boldsymbol{F}$ are defined in Eqs. (14) and (15).

From Eq. (12) or (16), the unknown $\boldsymbol{a}$ can be obtained. Then using the GFSA defined by Eq. (2), the potential field $u$ for all $x \in \bar{\Omega}$ can be attained.

\section{Numerical tests}

In this section, several numerical examples of $2 \mathrm{D}$ potential problems are presented to demonstrate the effectiveness of the proposed GMFS. In this section, the traditional MFS will be only considered for comparative analysis. In all of the examples in this section, the units of geometrical dimensions and field variables are assumed to be non-dimensional.

\subsection{A circular domain with Dirichlet BCs}

We first consider the Laplace equation in a circular domain with radius 1 and center at origin, and Dirichlet BCs is imposed on the boundary based to the following analytical solution:

$$
u(x, y)=\cos (x) \cosh (y)+\sin (x) \sinh (y) .
$$

Initially, we apply the GMFS to give a tentative solution for this problem. For numerical implementation, we use only 20 boundary nodes and adopt two nodal distribution scheme, uniformly distributed nodes and random nodes, as shown in Fig. 3. Then we solve it by the GMFS1 method with the given 20 boundary nodes, and show numerical results in Fig. 4. We observe that, the GMFS method can get good solution with a small number of boundary nodes. 

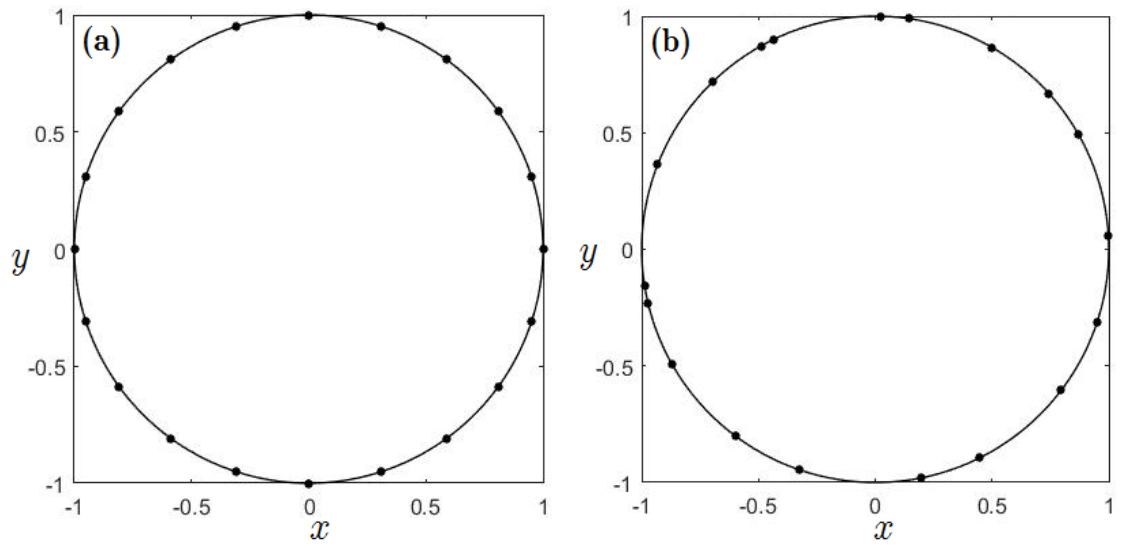

Fig. 3. Nodal distribution for the circular domain problem: (a) regular nodes; (b) random nodes.
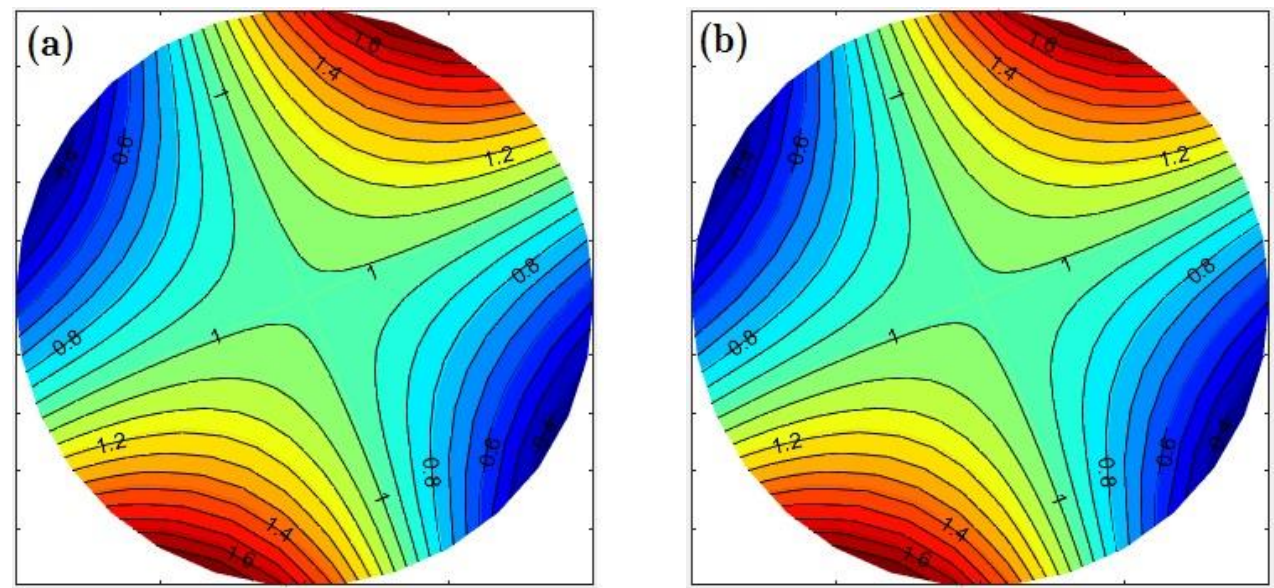

Fig. 4. Solution results on domain for the circular domain problem: (a) numerical results; (b) analytical results.

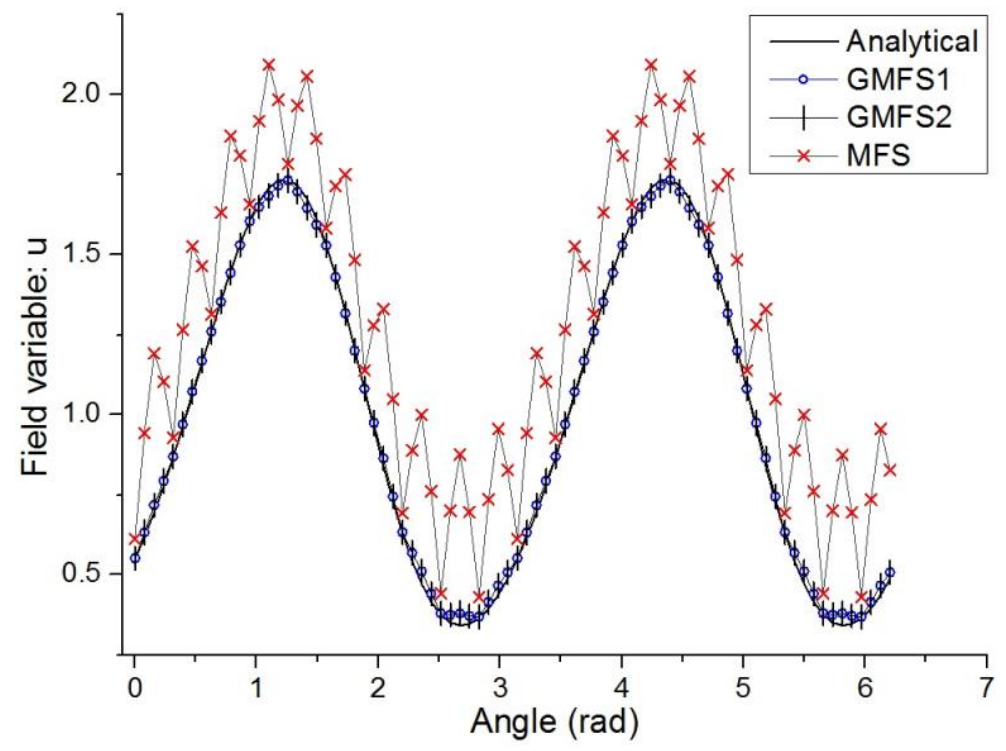

Fig. 5. Comparing numerical solutions on an analytical path with a bad offset for the circular domain problem.

Next, we test the numerical stability of the GMFS. In tentative computation, the MFS is inaccurate when the offset is near to the boundary. Then we use $d=0.07 \bar{R}$ for the MFS. And we embed the bad offset in the offsets of the GMFS, as $\left\{d_{\mathrm{p}}\right\}=\{0.07,0.5,0.7,0.9,1.2\} \bar{R}$. 
Furthermore, we solve the problem with the 20 uniformly distributed boundary nodes, and give the results on a circular analytical path of $P=0.99 R$ with 80 measuring points, as shown in Fig. 5. It can be seen that the MFS has failed to achieve reasonable solution, but the GMFS is still stable, when the bad offset is used in both.

Finally, we test the numerical convergence of the GMFS. We choose a circle of $P=0.5 R$ as the analytical path, and use 80 test points to evaluate the numerical errors. An average relative error is defined as

$$
E r=\frac{1}{m} \sqrt{\sum_{k=1}^{m}\left(\xi_{k}^{\text {num }}-\xi_{k}^{\text {ana }}\right)^{2} / \sum_{k=1}^{m}\left(\xi_{k}^{\text {ana }}\right)^{2}}
$$

where $m$ is the number of the test points. Let $\xi_{k}^{\text {num }}$ and $\xi_{k}^{\text {ana }}$ denote the numerical and analytical solution at the $k$-th test point, respectively. To compare the accuracy, we select the average mid-value of the GMFS' offsets $\left\{d_{\mathrm{p}}\right\}$ as the MFS' offset $d$; i.e., $d=\left(\max \left\{d_{p}\right\}-\min \left\{d_{p}\right\}\right) / 2$.

In the comparison test, both the uniform-node scheme and random-node scheme are considered, and the results are shown in Fig. 6. It can be seen that the GMFS is highly accurate in this problem. But, the GMFS doesn't show a distinct advantage in terms of accuracy and convergence when comparing to the MFS. In particular, GMFS1 is less accurate using randomnode scheme. It is easy to see that, in this example, the MFS gets a good offset value, but the values of the GMFS's offsets are not so good.

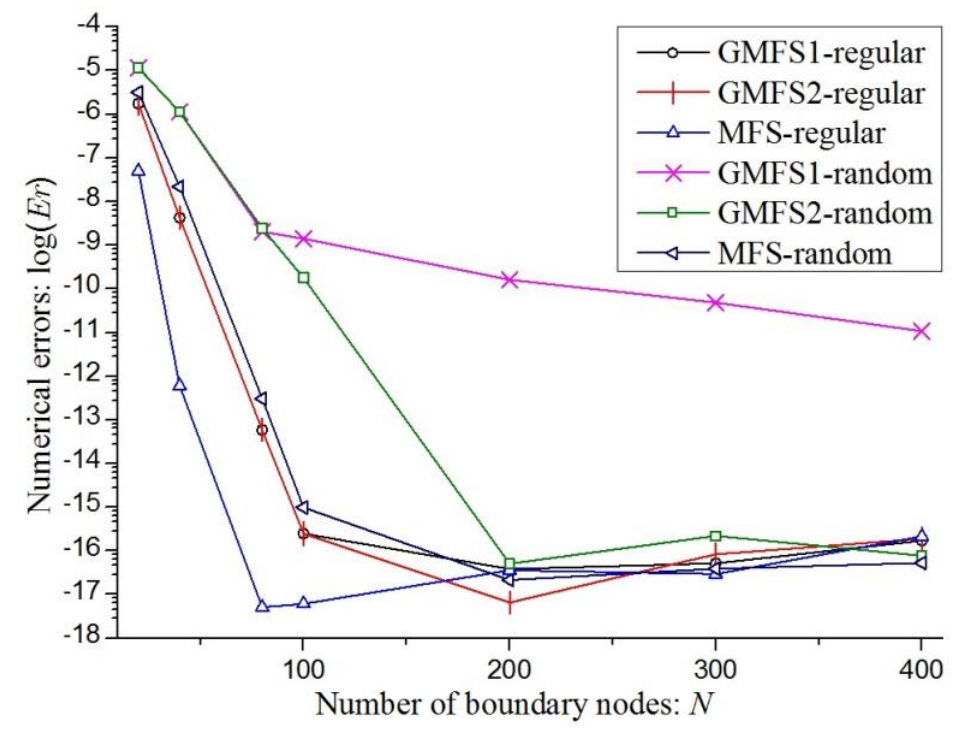

Fig. 6. Comparing numerical errors for the circular domain problem.

\subsection{An epitrochoid domain with mixed BCs}

Next, the Laplace equation for an epitrochoid domain with mixed Dirichlet and Neumann 
BCs is considered. The parametric equation of epitrochoid boundary is defined as

$$
\rho(\theta)=\sqrt{(a+b)^{2}+1-2(a+b) \cos (a \theta / b)}
$$

where the shape parameters are taken as $a=3, b=1$. The upper boundary of $0 \leq \theta \leq \pi$ is imposed as Dirichlet condition while $\pi<\theta<2 \pi$ is imposed as Neumann condition. The analytical solution is given by:

$$
u(x, y)=\exp (x) \cos (y), \quad(x, y) \in \bar{\Omega} .
$$

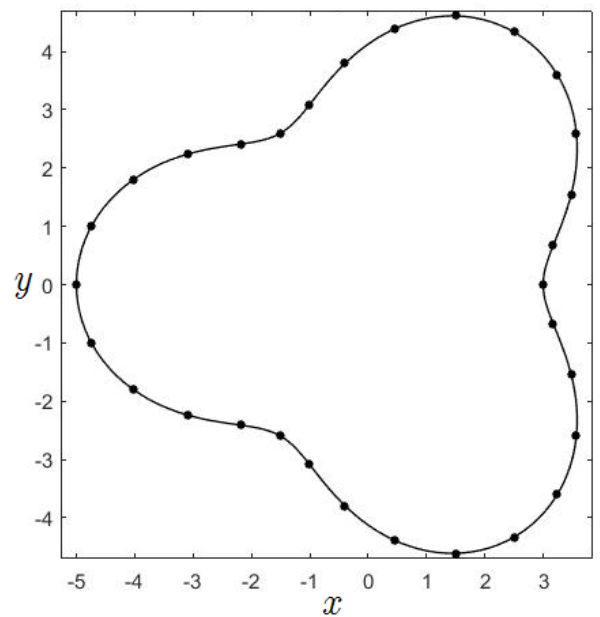

Fig. 7. Nodal distribution for the epitrochoid domain problem.

Initially, we will use the GMFS with 30 boundary nodes to give a tentative solution. The boundary nodes are shown in Fig. 7, and the numerical and analytical results are shown in Fig. 8. Obviously, the GMFS method can give quite reasonable solution with small number of boundary nodes.
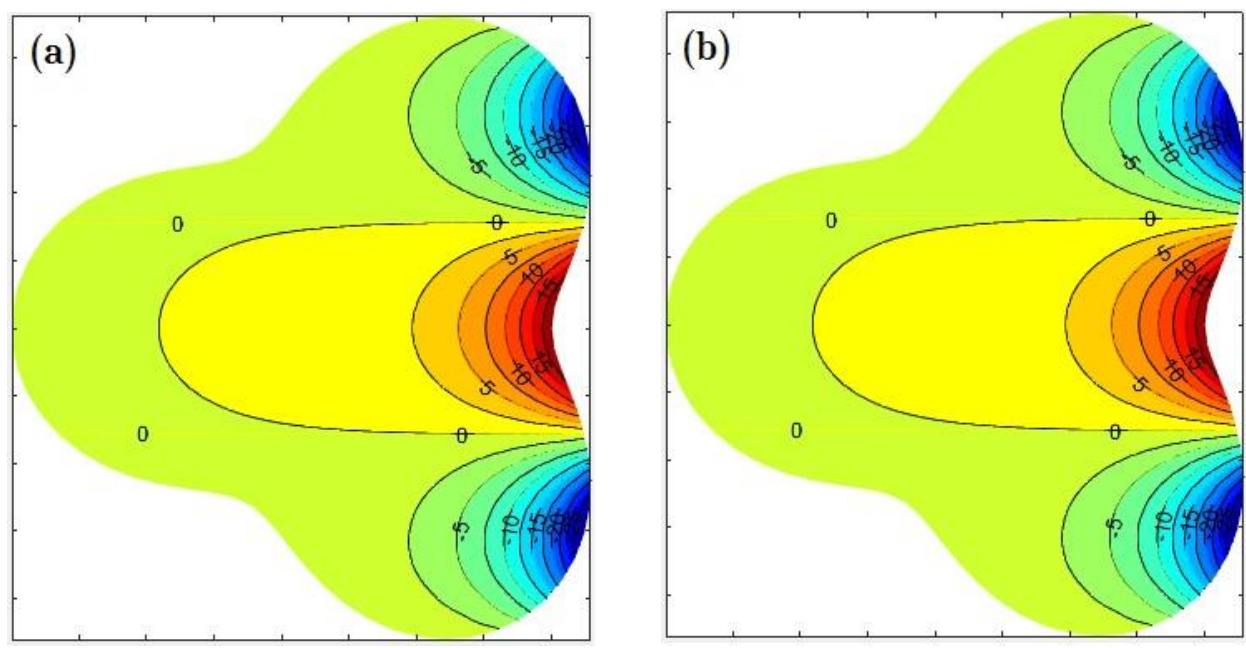

Fig. 8. Solution results on domain for the epitrochoid domain problem: (a) numerical results; (b) analytical results.

Next, we test the numerical stability. Since the condition number of the GMFS' system matrix is mainly effected by the max value of its offsets, and then we take the max value $d_{m}$ as 
the MFS' offset, and give a comparing solution. Moreover, since the condition number is fluctuating with the number of the boundary nodes $N$, so $N=100$ and $N=200$ are both considered in the comparing solution. The results are shown in Fig. 9. We observe that the GMFS always attains a better condition number comparing with the MFS, while the max offset value is used in both methods. Therefore, we can say that the GMFS is more stable than the MFS in terms of "condition number". In addition, we find an interesting phenomenon: GMFS1's condition number is better than that of GMFS2's for denser nodes ( $N=200)$, but it is opposite for sparser nodes $(N=100)$. This is an indication that the GMFS1 is preferable, but not always.

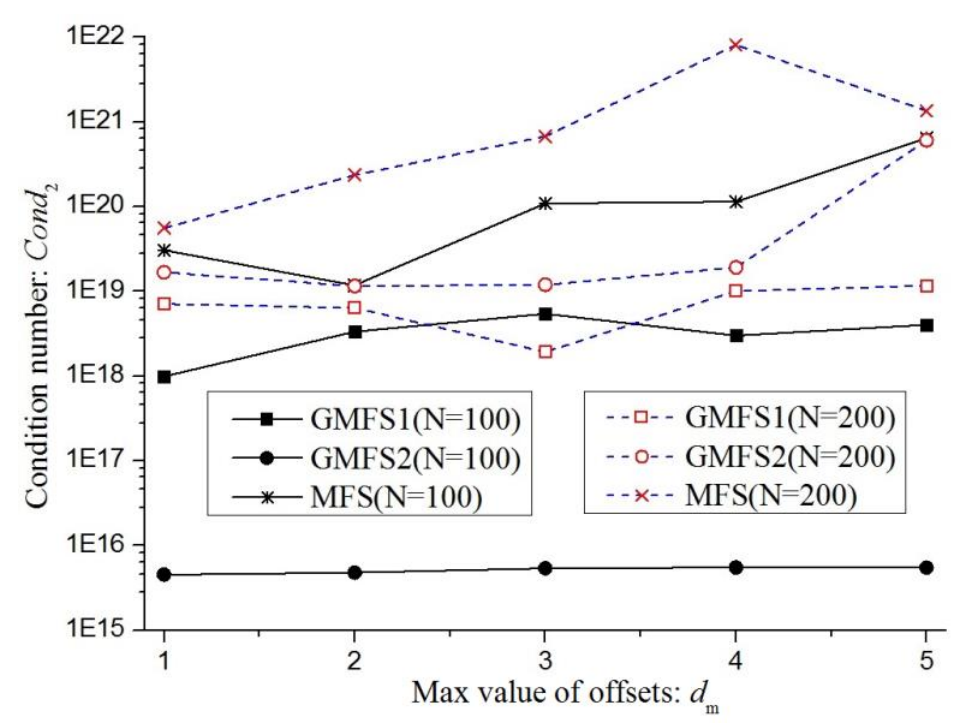

Fig. 9. Comparing condition numbers of the system matrix for the epitrochoid domain problem.

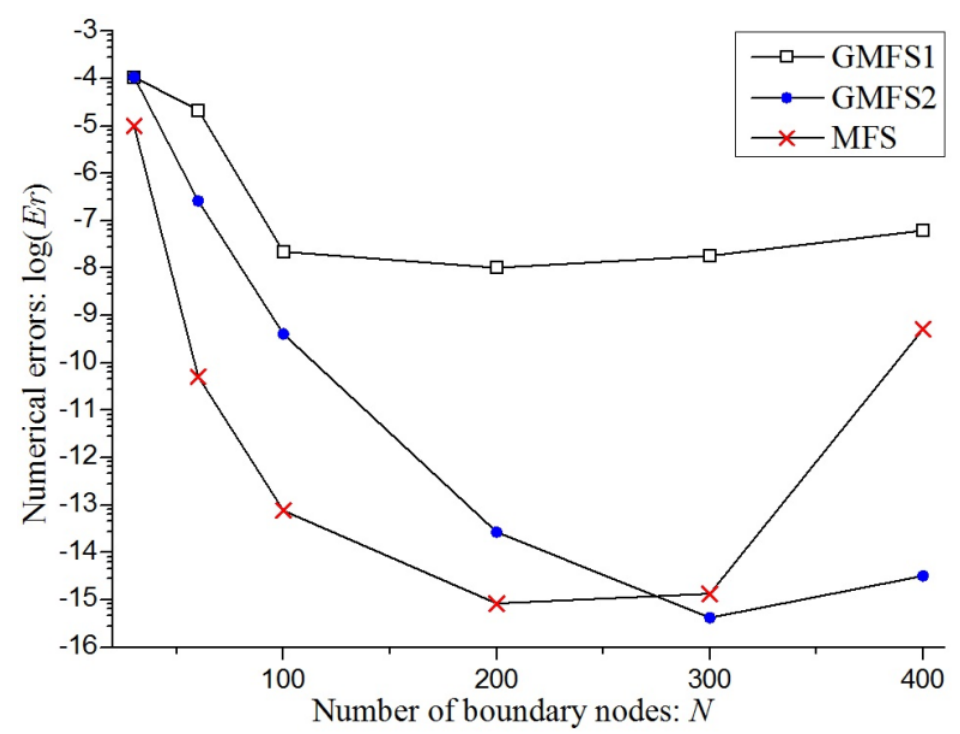

Fig. 10. Comparing numerical errors for the epitrochoid domain problem.

Additionally, we test the numerical convergence. We choose an analytical path as $P=0.9$ $\rho$, and use 80 test points to evaluate the numerical errors. Similarly, we select the average mid 
value of the GMFS' offsets $\left\{d_{\mathrm{p}}\right\}$ as the MFS' offset. The results are shown in Fig. 10. It can be seen that, the GMFS could be highly accurate, and the GMFS2 is clearly superior than the GMFS1. Based on this and previous tests, GMFS2 is the method of choice. Moreover, the MFS with an appropriate offset shows good accuracy and convergence, but, its instability is evidently observed with $N>300$ in this test.

Finally, we test the effectiveness of the diffuse density. For a stationary size /range of the GMFS' offsets, choosing as (0.1 0.5), we use an interval $h_{p}$ to diffuse, as $\left\{d_{\mathrm{p}}\right\}=\left(0.1: h_{p}: 0.5\right) \bar{R}$. We choose $h_{p}=0.1,0.05,0.01,0.005,0.001$, which denotes the diffuse density. The numerical errors are shown in Fig. 11, on the analytical path $P=0.9 \rho$ with the 80 test points. Number of the boundary nodes $N=60$ and 120 are both considered in the comparing solution. It shows that the GMFS has a diffuse-density convergence.

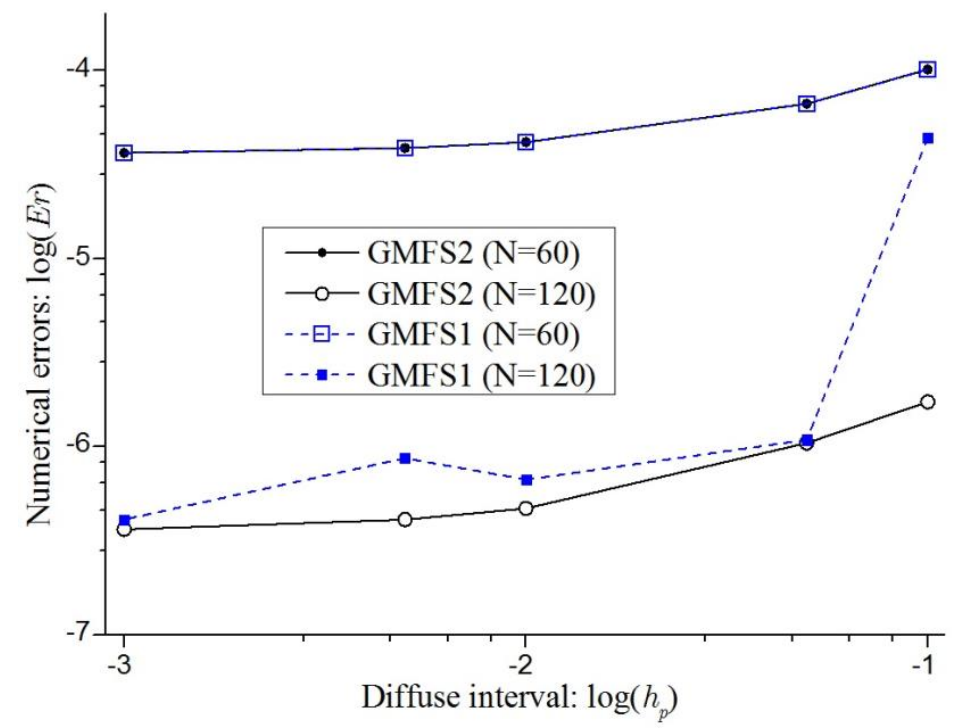

Fig. 11. Numerical errors with the diffuse interval for the epitrochoid domain problem.

\subsection{An amoeba-like domain with multi boundaries and mixed BCs}

An amoeba-like domain with multi-boundaries and mixed Dirichlet and Neumann boundary conditions is considered. The inner boundary is a circle with $r=0.2$ and center $(x, y)=(0.5,0.5)$. The circular boundary is imposed with Dirichlet condition. The outer amoeba-like boundary is imposed Neumann condition. The parametric equation of the amoeba-like boundary is defined as follows:

$$
\rho(\theta)=\exp (\sin (\theta)) \sin ^{2}(2 \theta)+\exp (\cos (\theta)) \cos ^{2}(2 \theta),
$$

which is shown in Fig. 12. The analytical solution on the domain is given as

$$
u(x, y)=\ln \sqrt{(x-0.5)^{2}+(y-0.5)^{2}}, \quad(x, y) \in \bar{\Omega} .
$$




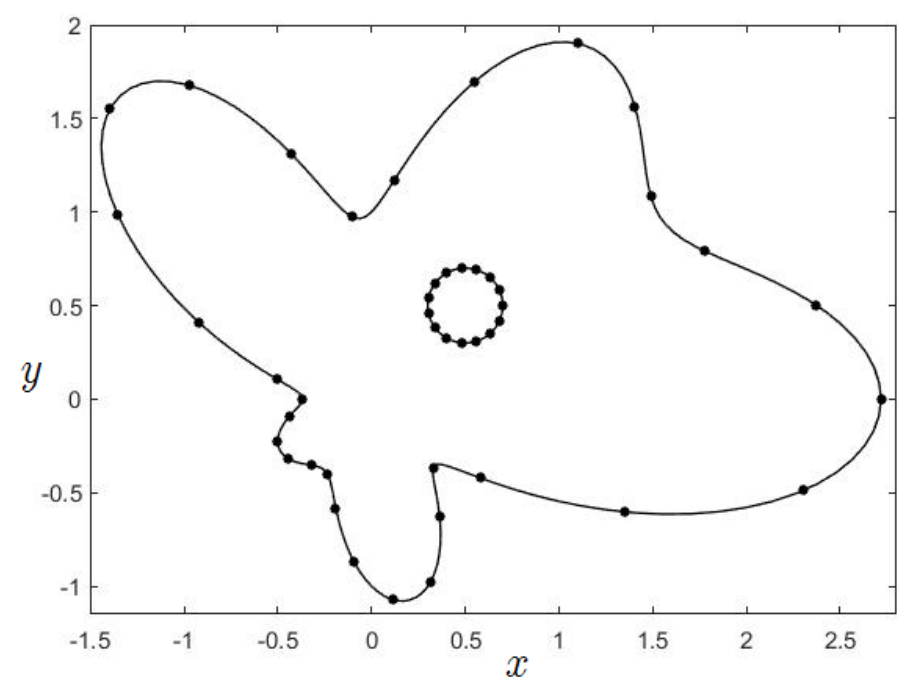

Fig. 12. Nodal distribution for the amoeba-like domain problem.

Initially, we use the GMFS method with 30 boundary nodes to give a tentative solution for this problem. The distribution of boundary nodes is shown in Fig. 12, and the numerical and analytical results on the domain are shown in Fig.13. Obviously, the GMFS method can give quite reasonable solution with small number of boundary nodes.
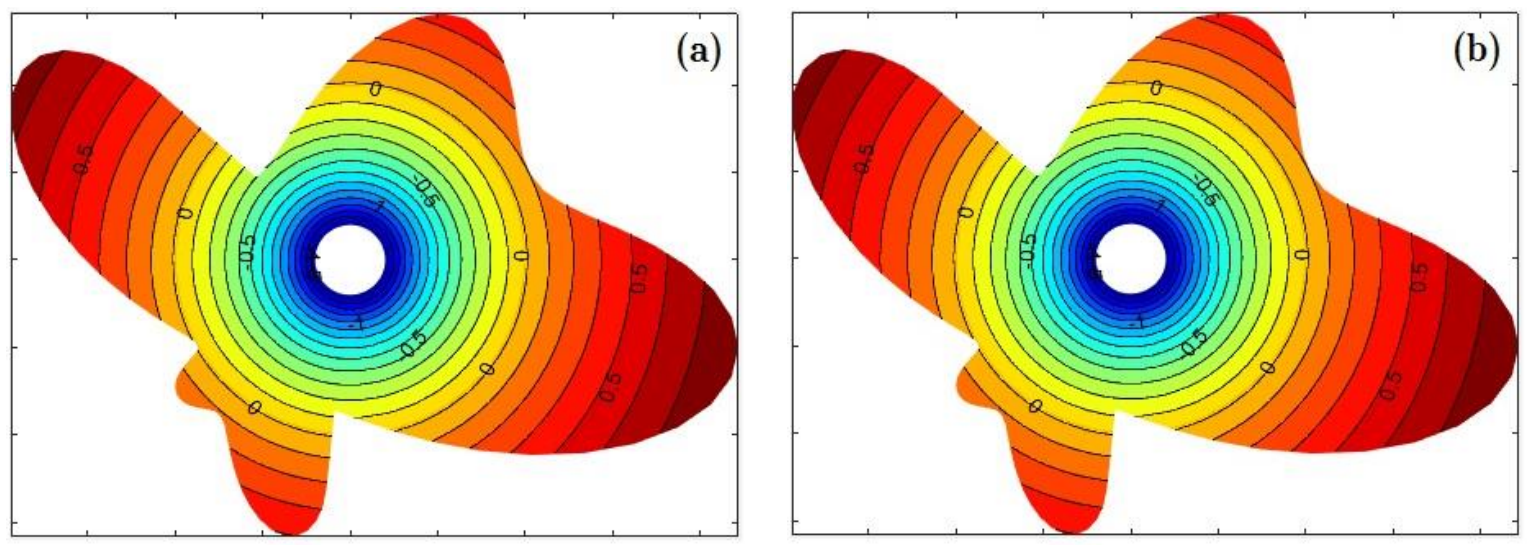

Fig. 13. Solution results on domain for the amoeba-like domain problem: (a) numerical results; (b) analytical results.

Next, we will test the numerical stability. For the MFS, we use a bad outset value of $d=0.001$, then embed the bad value in the GMFS' offsets. We solve the problem with the initial 30 boundary nodes, and give the results on an analytical path of $P=0.5 \rho$ with 80 measuring points, as shown in Fig. 14. It can be seen that the MFS is obviously inaccurate, but the GMFS is still accurate. This further illustrates that the GMFS is more stable than the MFS.

Finally, we will test the numerical convergence. We choice an analytical path as $P=0.5 \rho$, and use 80 measuring points to evaluate the numerical errors. Similarly, we select the average mid value of the GMFS' offsets as the MFS' offset. The results are shown in Fig. 15. It can be seen that, the GMFS2 shows best convergence, but GMFS1 shows rather lower convergence. 


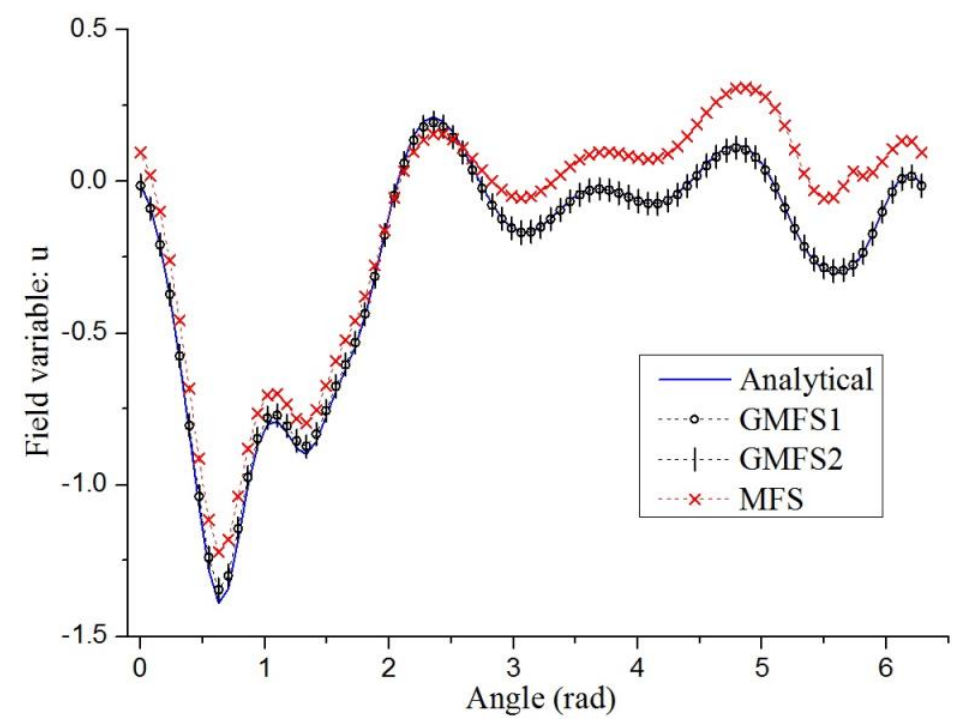

Fig. 14. Comparing numerical solutions with a bad offset for the amoeba-like domain problem.

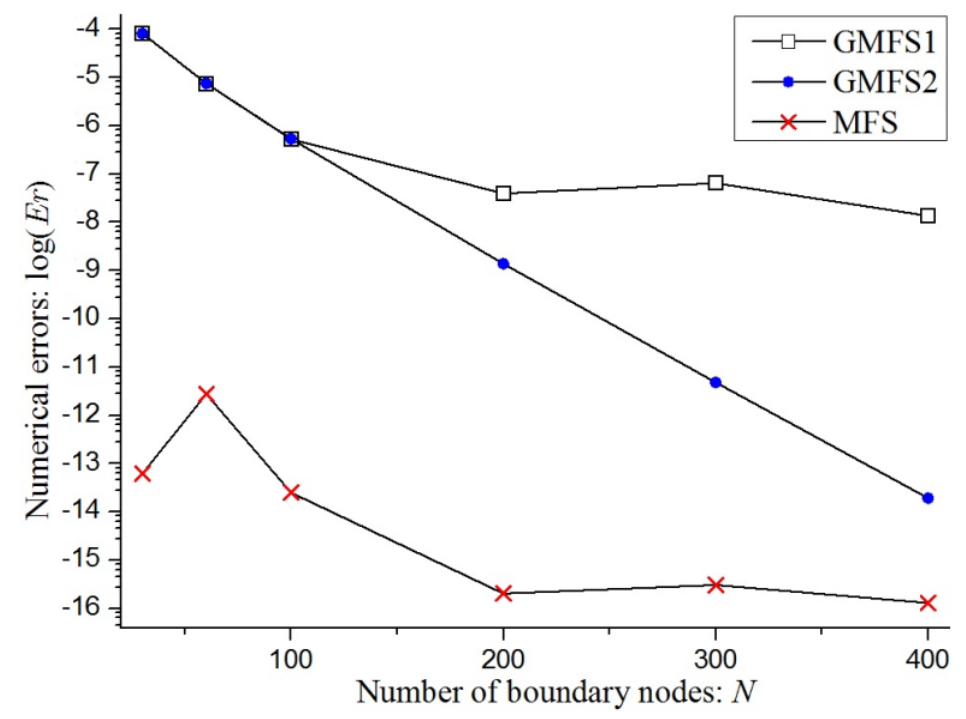

Fig. 15. Comparing numerical errors for the amoeba-like domain problem.

\subsection{A biharmonic problem with Robin BCs}

Consider a biharmonic problem as an extended potential problem, which is defined as

$$
\nabla^{4} u(\boldsymbol{x}) \equiv \nabla^{2}\left(\nabla^{2} u\right)=0, \quad \boldsymbol{x} \in \Omega,
$$

subject to the Robin BCs:

$$
u(\boldsymbol{x})=\bar{u}(\boldsymbol{x}), \quad \nabla^{2} u(\boldsymbol{x})=\bar{Q}(\boldsymbol{x}), \quad \boldsymbol{x} \in \Gamma,
$$

or

$$
u(\boldsymbol{x})=\bar{u}(\boldsymbol{x}), \quad u_{, n}(\boldsymbol{x})=\bar{q}(\boldsymbol{x}), \quad \boldsymbol{x} \in \Gamma,
$$

and the field is defined in a L-shaped domain as shown in Fig. 16. The boundary condition $\bar{u}$ is given as: 


$$
\bar{u}(x, y)=x^{2} y^{3}
$$

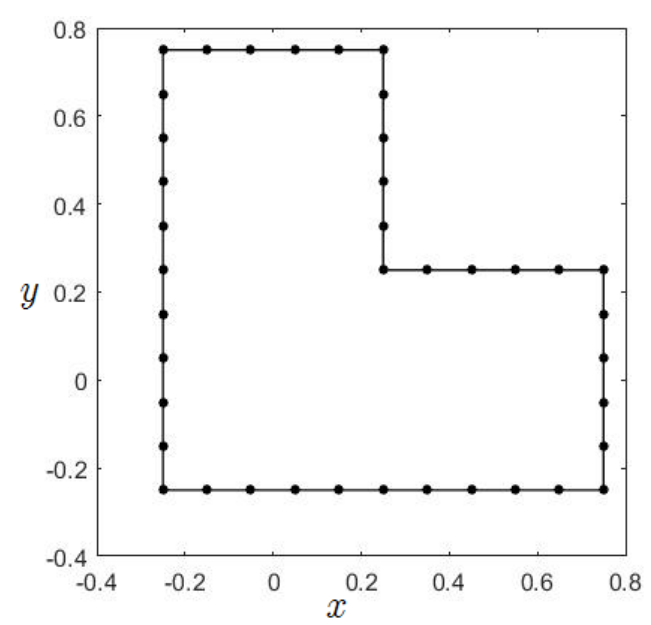

Fig. 16. Nodal distribution for the biharmonic problem.

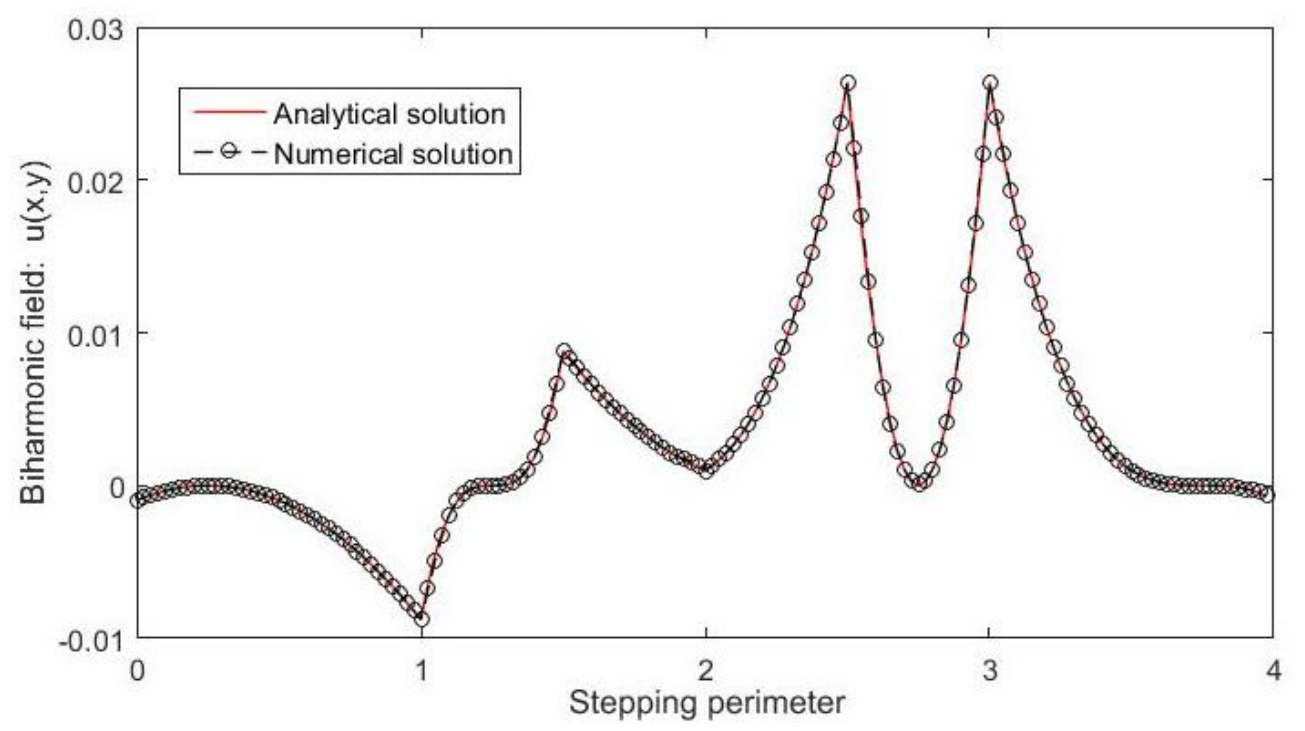

Fig. 17. Solutions on the analytical path for the biharmonic problem.

and $\bar{Q}=\nabla^{2} \bar{u}$, or $\bar{q}=\bar{u}_{, n}$ is correspondingly used. Note that the exact solution is not available in this example. For solving the problem, some special technique should be adopted, detailed in [19, 41]. Based on the Maximum Principle [19, 41-43], the maximum error will occur on the boundary. Hence, we can measure the error on the boundary where the boundary condition is known. We solve the problem with 40 boundary nodes, as shown in Fig. 16, and only the GFMS2 is used. We choose an analytical path "stepping perimeter" as that, beginning at the left-bottom-corner point and stepping forward to a counterclockwise direction on the boundary. Then we give the numerical results with 160 measuring points in the Fig. $17\left(\left\{d_{\mathrm{p}}\right\}=(0.02 \sim 0.1)\right.$ $\bar{R}$, Eq.(24) BCs).

Next, we give a comparing of numerical errors for the GMFS and the MFS. Using the BCs 
by Eq. (24), and a 0.01 interval for $\left\{d_{\mathrm{p}}\right\}$ diffused, and taking the mid value of $\left\{d_{\mathrm{p}}\right\}$ as the offset for the MFS, then the results are shown in Tab. 1. Using the BCs by Eq.(25), and a 0.001 interval for $\left\{d_{\mathrm{p}}\right\}$ diffused, and the mid value of $\left\{d_{\mathrm{p}}\right\}$ for the MFS, then the results are shown in Tab. 2 . Evidently, the GMFS is stable and accurate, but the MFS is unstable and less accurate. In addition, we noticed that the GMFS showed a convergence with increasing range (diffuse-size convergence) in this computation. This is another advantage for the GMFS.

Tab. 1. Comparing of numerical errors (Er) for GMFS and MFS: Eq.(24) BCs

\begin{tabular}{ccc}
\hline Range of $\left\{d_{\mathrm{p}}\right\}$ & GMFS & MFS \\
\hline$(0.01 \sim 0.1) \bar{R}$ & $1.0777 \mathrm{e}-04$ & $1.1608 \mathrm{e}-04$ \\
$(0.01 \sim 0.2) \bar{R}$ & $9.6753 \mathrm{e}-05$ & $1.0908 \mathrm{e}-04$ \\
$(0.01 \sim 0.3) \bar{R}$ & $8.9717 \mathrm{e}-05$ & $1.0606 \mathrm{e}-04$ \\
$(0.01 \sim 0.4) \bar{R}$ & $8.4983 \mathrm{e}-05$ & collapsed (warning) \\
$(0.01 \sim 0.5) \bar{R}$ & $8.1330 \mathrm{e}-05$ & $9.7136 \mathrm{e}-04$ \\
\hline
\end{tabular}

Tab. 2. Comparing of numerical errors (Er) for GMFS and MFS: Eq.(25) BCs

\begin{tabular}{ccc}
\hline Range of $\left\{d_{\mathrm{p}}\right\}$ & GMFS & MFS \\
\hline$(0.001 \sim 0.1) \bar{R}$ & $1.4784 \mathrm{e}-04$ & $4.6663 \mathrm{e}-04$ \\
$(0.001 \sim 0.2) \bar{R}$ & $1.2072 \mathrm{e}-04$ & $1.5907 \mathrm{e}-04$ \\
$(0.001 \sim 0.3) \bar{R}$ & $1.0564 \mathrm{e}-04$ & $2.2991 \mathrm{e}-04$ \\
$(0.001 \sim 0.4) \bar{R}$ & $9.6537 \mathrm{e}-05$ & 1.0964 (warning) \\
$(0.001 \sim 0.5) \bar{R}$ & $9.0233 \mathrm{e}-05$ & $6.3016 \mathrm{e}-04$ \\
\hline
\end{tabular}

\section{Conclusions}

In our opinion, the real problem of the MFS is that: the success and failure for the solution are entirely depending on a single offset for a target node. That will put the method in an unstable state, and even in a crisis of collapse. The GMFS uses the GFSA to approximate, which blasts the source node into an intervention-point diffuse (IPD). Though the offset concept still exists, the solution performance of the GMFS is decided by a series of offsets, rather than the single one. That helps the GMFS being more stable and better convergence, and supports the method showing the diffuse-density convergence and diffuse-size convergence. Moreover, this improvement is simple and cheap, and no other problems apparently surfacing. Theoretically, there are few barriers for the GMFS to be applied to other common boundary value problems, only if the fundamental solution is available.

There are 2 algorithms presented for the GMFS, GMFS1 and GMFS2. We had expected that the GMFS1 to be more stable, but we do have the numerical result to support such 
expectation. Hence, we would like to recommend the GMFS2 as the standard algorithm here. But, the further examination of the GMFS1 is still open. Moreover, we have given a preliminary standard offsets for the GMFS, as in the Eqs. (4) and (5). But, it should be tested and corrected further. In addition, a regional IPD, as shown in Fig. 1(a), is another option for the GMFS, its effect is also a topic for further research.

\section{Acknowledgements}

The authors would like to thank the support from the National Natural Science Foundation of China (No.51478053), and Open Fund of Key Laboratory of Road Structure and Material of Ministry of Transport: CSUST (KFJ120201).

\section{References}

[1] Fairweather G, Karageorghis A. The method of fundamental solutions for elliptic boundary value problems[J]. Advances in Computational Mathematics, 1998, 9(1-2): 69-95.

[2] Fairweather G, Karageorghis A, Martin P A. The method of fundamental solutions for scattering and radiation problems[J]. Engineering Analysis with Boundary Elements, 2003, 27(7): 759-769.

[3] Golberg M A. The method of fundamental solutions for Poisson's equation[J]. Engineering Analysis with Boundary Elements, 1995, 16(3): 205-213.

[4] Golberg M A, Chen C S. The method of fundamental solutions for potential, Helmholtz and diffusion problems[J]. Boundary integral methods-numerical and mathematical aspects, 1998: 103-176.

[5] Chen, C. S., Karageorghis, A., \& Smyrlis, Y. S. (Eds.). The method of fundamental solutions: a meshless method. Atlanta: Dynamic Publishers, 2008.

[6] Lv H, Hao F, Wang Y, et al. The MFS versus the Trefftz method for the Laplace equation in 3D[J]. Engineering Analysis with Boundary Elements, 2017, 83: 133-140.

[7] Chen W, Fu Z J, Chen C S. Recent advances in radial basis function collocation methods[M]. Berlin: Springer, 2014.

[8] Belytschko T, Krougauz Y, Organ D, Fleming M, Krysl P. Meshless method: an overview and recent developments. Comput. Methods Appl. Mech. Engrg., 1996, 139:3 47.

[9] Chen J S, Hillman M, Chi S W. Meshfree methods: progress made after 20 years[J]. Journal of Engineering Mechanics, 2017, 143(4): 04017001.

[10] Mukherjee Y X, Mukherjee S. The boundary node method for potential problems[J]. International Journal for Numerical Methods in Engineering, 1997, 40(5): 797-815.

[11] Zhu T, Zhang J D, Atluri S N. A local boundary integral equation (LBIE) method in computational mechanics, and a meshless discretization approach[J]. Computational mechanics, 1998, 21(3): 223-235.

[12] Zhang J, Yao Z, Li H. A hybrid boundary node method[J]. International Journal for Numerical Methods in Engineering, 2002, 53(4): 751-763.

[13] Li G, Aluru N R. Boundary cloud method: a combined scattered point/boundary integral approach for boundary-only analysis[J]. Computer Methods in Applied Mechanics and Engineering, 2002, 191(2122): $2337-2370$.

[14] Zhang J, Qin X, Han X, et al. A boundary face method for potential problems in three dimensions[J]. International Journal for Numerical Methods in Engineering, 2009, 80(3):320-337.

[15] Katsurada M, Okamoto H. The collocation points of the fundamental solution method for the potential 
problem[J]. Computers \& Mathematics with Applications, 1996, 31(1): 123-137.

[16] Alves C J S. On the choice of source points in the method of fundamental solutions[J]. Engineering Analysis with Boundary Elements, 2009, 33(12): 1348-1361.

[17] Cisilino A P, Sensale B. Optimal placement of the source points for singular problems in the method of fundamental solutions, Advances in Boundary Element Techniques II Denda, M[J]. Hoggar, Geneva, 2001.

[18] Cisilino A P, Sensale B. Application of a simulated annealing algorithm in the optimal placement of the source points in the method of the fundamental solutions[J]. Computational mechanics, 2002, 28(2): 129-136.

[19] Chen C S, Karageorghis A, Li Y. On choosing the location of the sources in the MFS[J]. Numerical Algorithms, 2016, 72(1): 107-130.

[20] Li M, Chen C S, Karageorghis A. The MFS for the solution of harmonic boundary value problems with non-harmonic boundary conditions[J]. Computers \& Mathematics with Applications, 2013, 66(11): $2400-2424$.

[21] Chen W. Symmetric boundary knot method[J]. Engineering Analysis with Boundary Elements, 2002, 26(6): 489-494.

[22] Hon Y C, Chen W. Boundary knot method for 2D and 3D Helmholtz and convection-diffusion problems under complicated geometry[J]. International Journal for Numerical Methods in Engineering, 2003, 56(13): 1931-1948

[23] Chen W, Hon Y C. Numerical investigation on convergence of boundary knot method in the analysis of homogeneous Helmholtz, modified Helmholtz, and convection-diffusion problems[J]. Computer methods in applied mechanics and engineering, 2003, 192(15): 1859-1875.

[24] Chen J T, Chang M H, Chen K H, et al. The boundary collocation method with meshless concept for acoustic eigenanalysis of two-dimensional cavities using radial basis function[J]. Journal of Sound and Vibration, 2002, 257(4): 667-711.

[25] Chen J T, Chang M H, Chen K H, et al. Boundary collocation method for acoustic eigenanalysis of three-dimensional cavities using radial basis function[J]. Computational Mechanics, 2002, 29(4-5): 392408.

[26] Young D L, Chen K H, Lee C W. Novel meshless method for solving the potential problems with arbitrary domain[J]. Journal of Computational Physics, 2005, 209(1):290-321.

[27] Young D L, Chen K H, Chen J T, et al. A modified method of fundamental solutions with source on the boundary for solving Laplace equations with circular and arbitrary domains[J]. Computer Modeling in Engineering and Sciences, 2007, 19(3): 197-221.

[28] Chen W. Singular boundary method: a novel, simple, meshfree, boundary collocation numerical method[J]. Chinese Journal of Solid Mechanics, 2009, 30(6): 592-599.

[29] Chen W, Fu Z J. A novel numerical method for infinite domain potential problems. Chinese Sci Bull, 2010, 55: $1598-1603$.

[30] Chen W, Wang F Z. A method of fundamental solutions without fictitious boundary[J]. Engineering Analysis with Boundary Elements, 2010, 34(5): 530-532.

[31] Qu W, Chen W, Zheng C. Diagonal form fast multipole singular boundary method applied to the solution of high - frequency acoustic radiation and scattering[J]. International Journal for Numerical Methods in Engineering, 2017, 111(9): 803-815.

[32] Li J, Chen W. A modified singular boundary method for three-dimensional high frequency acoustic wave problems[J]. Applied Mathematical Modelling, 2018, 54: 189-201.

[33] Liu Y J. A new boundary meshfree method with distributed sources[J]. Engineering Analysis with Boundary Elements, 2010, 34(11):914-919.

[34] Kim S. An improved boundary distributed source method for two-dimensional Laplace equations[J]. 
Engineering Analysis with Boundary Elements, 2013, 37(7): 997-1003.

[35] Khambampati A K, Lee Y G, Kim K Y, et al. A meshless improved boundary distributed source method for two-phase flow monitoring using electrical resistance tomography[J]. Engineering Analysis with Boundary Elements, 2015, 52: 1-15.

[36] Kupradze V D, Aleksidze M A. The method of functional equations for the approximate solution of certain boundary value problems[J]. USSR Computational Mathematics \& Mathematical Physics, 1964, $4(4): 82-126$.

[37] Kupradze V D. A method for the approximate solution of limiting problems in mathematical physics[J]. USSR Computational Mathematics and Mathematical Physics, 1964, 4(6): 199-205.

[38] Kupradze V D. On the approximate solution of problems in mathematical physics[J]. Russian Mathematical Surveys, 1967, 22(2): 58-108.

[39] Wen P H. Point intensity method of solving circular plate resting on elastical subgrade[J]. Engineering Mechanics, 1987, 4(2): 18-26.

[40] Yang J J, Zheng J L. Intervention-point principle of meshless method[J]. Chinese Science Bulletin, 2013, 58(4-5): 478-485.

[41] Karageorghis A, Fairweather G. The method of fundamental solutions for the numerical solution of the biharmonic equation[J]. Journal of Computational Physics, 1987, 69(2): 434-459.

[42] Poullikkas A, Karageorghis A, Georgiou G. Methods of fundamental solutions for harmonic and biharmonic boundary value problems[J]. Computational Mechanics, 1998, 21(4-5): 416-423.

[43] Pei X, Chen C S, Dou F. The MFS and MAFS for solving Laplace and biharmonic equations[J]. Engineering Analysis with Boundary Elements, 2017, 80: 87-93. 